\title{
Neurotoxic effects of acrylamide on dopaminergic neurons in primary mesencephalic cell culture
}

\author{
Khaled Radad ${ }^{1}$, Mubarak Al-Shraim ${ }^{1}$, Ahmed Al-Emam ${ }^{1,2}$, Rudolf Moldzio ${ }^{3}$, Wolf-Dieter Rausch ${ }^{3}$ \\ ${ }^{1}$ Department of Pathology, College of Medicine, King Khalid University, Abha, Saudi Arabia, ${ }^{2}$ Department of Forensic Medicine and \\ Clinical Toxicology, Faculty of Medicine, Mansoura University, Egypt, ${ }^{3}$ Department for Biomedical Sciences, University of Veterinary \\ Medicine, Vienna, Austria
}

\begin{abstract}
Introduction: Exposure to acrylamide is increasing worldwide as a result of its heavy use in industry and formation in carbohydrate-rich food cooked at high temperature. Despite its neurotoxicity, no studies have shown its toxic effects on dopaminergic neurons yet. Therefore, the current study was carried out to show whether acrylamide adversely affects primary cultured dopaminergic neurons.

Material and methods: Acrylamide (0.001, 0.01, 0.1, 1, 2 mM) was added to two different groups of primary mesencephalic cell cultures on the $9^{\text {th }}$ day in vitro for 24 and $48 \mathrm{~h}$, respectively. Moreover, a group of cultures was treated with lower concentrations of acrylamide $(0.01,0.05,0.1,0.5 \mathrm{mM})$ on the $6^{\text {th }}$ day in vitro for 5 consecutive days to investigate its long-term effects on dopaminergic neurons. Following each treatment, culture media were obtained for measuring lactate dehydrogenase, and cultured cells were stained immunocytochemically against tyrosine hydroxylase and neuronal nuclear antigens.

Results: Treatment of cultures with acrylamide for $48 \mathrm{~h}$ significantly reduced the number of dopaminergic neurons, adversely altered the morphology of the surviving neurons and increased levels of lactate dehydrogenase in the culture media. Similar treatment of cultures with acrylamide also resulted in lower numbers of total neuronal cells as shown by a reduced expression of the neuronal nuclear antigen. Prolonged treatment of cultures with lower concentrations of acrylamide slightly reduced the survival of dopaminergic neurons but increased the release of lactate dehydrogenase into the culture media as well.

Conclusions: The current study shows, for the first time, neurotoxicity of acrylamide on dopaminergic neurons in the primary mesencephalic cell culture.
\end{abstract}

Key words: acrylamide, dopaminergic neurons, neurotoxicity, neuronal nuclear antigen, Parkinson's disease.

\section{Introduction}

Acrylamide (ACR) is a chemical that used widely in science, industry and technology [14]. In 2002, the Swedish National Food Agency and Stockholm University discovered that ACR can naturally form in many starchy foods prepared at high tempera- ture such as biscuits, cereals, potatoes crisps, French fries and coffee [2]. Acrylamide forms in foods as a result of interaction between sugars and an asparagine amino acid through Maillard reaction [24]. Scientists classified ACR as a well-known neurotoxin [3] and they reported that it can affect both central and peripheral nervous systems in human and

\section{Communicating author}

Prof. Khaled Radad, Department of Pathology, College of Medicine, King Khalid University, Abha, Saudi Arabia,

e-mail: khaledradad@hotmail.com 
experimental animals [18]. In this context, Pennisi et al. [18] reported that humans who were exposed subchronically to ACR showed muscle weakness, hand and feet numbness, ataxia and gait abnormalities. Semla et al. [25] observed that treatment of rats with ACR caused foot splay, ataxia and muscle weakness to paralysis of hind limbs. These neuronal disabilities were reported to result from inhibiting kinesin-based fast axonal transport, alteration of neurotransmitter levels and direct inhibition of neurotransmission [7]. A direct toxic effect of acrylamide was also reported on neuronal cells both in in vitro and in vivo. For example, Mehri et al. [16] showed that ACR decreased cell viability of PC12 cells, and Abdelall et al. [1] and Lai et al. [10] reported progressive degeneration of Purkinje cells and hippocampal neurons by ACR in rats, respectively.

Parkinson's disease (PD) is a chronic and progressive neurodegenerative disorder affecting about $1 \%$ of elderly people worldwide [9]. The selective loss of dopaminergic neurons in substantia nigra pars compacta (SNpc) and depletion of striatal dopamine are considered the apparent pathological processes leading to most of clinical signs in PD [6]. Apart of the genetic form of PD which affects about $5-10 \%$ of cases, scientists have only identified some risk factors that are associated with the occurrence of PD and failed so far to explain the aetiology underlying dopaminergic cell loss in the sporadic form $[15,26]$. Among these risk factors, environmental exposure to industrial chemicals, organic solvents, pesticides and heavy metals is common [19]. Although ACR was recognized as a well-known neurotoxin a long time ago, and dietary and occupational ACR exposure are significantly increasing nowadays, no studies have investigated its neurotoxic effects on dopaminergic neurons so far. Accordingly, the present study was built up to investigate whether ACR adversely affects dopaminergic neurons in primary mesencephalic cell culture, the most reliable in vitro model for PD.

\section{Material and methods \\ Preparation of primary mesencephalic cell cultures}

Care and handling of pregnant mice (OF1/SPF) were performed in accordance with the guidelines of the European Union Council (86/609/EU) for the use of laboratory animals. On gestation day 14 , pregnant mice were killed, the uteri were dissected and the embryos were carefully removed to a Petri dish containing Dulbecco's phosphate buffered saline (DPBS, Invitrogen, Germany, $\mathrm{pH}$ 7.2). Brains were then obtained, mesencephala were excised and cultures were prepared according to Radad et al. [20]. In brief, mesencephala were cleaned from meninges and cut into small pieces in a drop of DPBS. The tissues were then incubated in a water bath at $37^{\circ} \mathrm{C}$ for $7 \mathrm{~min}$ in presence of $0.25 \%$ trypsin solution $(2 \mathrm{ml}$ ) and $0.02 \%$ DNase I in HBSS (2 ml). After centrifugation at $100 \times \mathrm{g}$ for 4 min, the supernatant was aspirated and the tissue pellet was mechanically dissociated with a fire-polished Pasteur pipette. Then, suspended cells were grown in Dulbecco's Modified Eagle Medium (DMEM) provided with $10 \mathrm{mM}$ HEPES buffer, $4 \mathrm{mM}$ glutamine and $10 \%$ heat-inactivated foetal calf serum (FCS). On the $1^{\text {st }}$ and $3^{\text {rd }}$ day in vitro (DIV), the medium was exchanged with DMEM supplemented with FCS. On the $5^{\text {th }}$ DIV, the medium was exchanged with FCS-contained medium (50\%) and serumfree DMEM supplemented with $0.02 \mathrm{ml} \mathrm{B-27/ml}$ (50\%) (Invitrogen, Germany). Serum free DMEM supplemented with $0.02 \mathrm{ml} \mathrm{B}-27 / \mathrm{ml}$ was used from the $6^{\text {th }}$ DIV and was subsequently replaced every 2 day.

\section{Treatment of cultures with acrylamide}

A $10 \mathrm{mM}$ stock solution of ACR (Sigma Aldrich) was prepared by dissolving $0.7108 \mathrm{mg}$ of ACR (MW: 71.08) in $1 \mathrm{ml}$ distilled water. The final treatment concentrations were prepared in DEMEM. On the $9^{\text {th }}$ DIV, two groups of cultures were treated separately with different concentrations of ACR (0.001, $0.01,0.1,1,2 \mathrm{mM}$ ) for 24 and $48 \mathrm{~h}$ to investigate the effects of short-term treatment with ACR on dopaminergic neurons. The lethal concentration $\left(\mathrm{LC}_{50}\right)$ of ACR on dopaminergic neurons was determined. To investigate the long-term effects of ACR on dopaminergic neurons, a group of cultures was treated with lower concentrations of ACR $(0.01,0.05,0.1,0.5 \mathrm{mM})$ on the $6^{\text {th }}$ DIV for 5 consecutive days.

\section{Identification of dopaminergic neurons}

At the end of ACR treatments, culture media were removed, aliquoted and stored at $-70^{\circ} \mathrm{C}$ till measurement of lactate dehydrogenase. Cultured cells were then washed carefully with PBS and fixed in histochoice for $15 \mathrm{~min}$ at room temperature. Then, cells were permeabilized with $0.4 \%$ Triton-X for 30 min. Cultured cells were rinsed 3 times with PBS 
and non-specific binding sites were blocked by $5 \%$ horse serum (Vectastain ABC Elite kite) for 90 min. To visualize dopaminergic neurons (THir neurons), cultured cells were incubated with anti-TH antibody overnight at $4^{\circ} \mathrm{C}$, secondary antibody (Vectastain) and avidin-biotin-horseradish peroxidase complex (Vectastain) for $90 \mathrm{~min}$ at room temperature. The reaction product was developed in a solution of diaminobenzidine $(1.4 \mathrm{mM})$ in PBS containing $3.3 \mathrm{mM}$ hydrogen peroxide $\left(\mathrm{H}_{2} \mathrm{O}_{2}\right)$. Dopaminergic neurons were counted with a Nikon inverted microscope in 10 randomly selected fields per well at 10x magnification. Moreover, 50 dopaminergic neurons (10/ field) were randomly selected in 5 randomly selected fields/well and their primary neurites were counted.

\section{Measurement of lactate dehydrogenase}

Lactate dehydrogenase (LDH) release serves as a marker of cytotoxicity and cell death [11]. The cytotoxic detection kit was used to measure LDH in the culture media according to the instructions of the manufacturer. In brief, $\mathrm{NADH}+\mathrm{H}^{+}$produced from $\mathrm{NAD}^{+}$by $\mathrm{LDH}$ is transferred by diaphorase to the yellow tetrazolium salt 2-[4-iodophenyl]-3-[4-nitrophenyl]-5-phenyltetrazolium chloride (INT) resulting in red formazan formation. The latter was measured spectrophotometrically at $490 \mathrm{~nm}$ with a reference wave length at $688 \mathrm{~nm}$. Supplemented DMEM was used as a blank and deducted as background.

\section{Neuronal nuclear antigen immunostaining}

To investigate the effect of ACR on the total neuronal cell populations, a set of cultures was administered with different concentrations of ACR (0.001, $0.01,0.1,1,2 \mathrm{mM}$ ) on the $9^{\text {th }}$ DIV for $48 \mathrm{~h}$. On the $11^{\text {th }}$ DIV, culture media were aspirated and cultured cells were immunohistochemically stained with antiNeuN (neuronal nuclear antigen) antibody (Chemicon, USA) for visualizing postmitotic neuronal cell types. In the cells, similar staining procedures were performed as described for anti-TH immunostaining except that the anti-TH was replaced with the antiNeuN antibody. NeuN immunoreactivity was quantitatively assessed in 10 photo/treatment condition using Adobe Photoshop software ${ }^{\circledR}$. In brief, the original photos were converted into black-white ones by the threshold tool. Density of black colour was mea- sured and presented as the percent of the average control [18].

\section{Statistics}

Data for each parameter were obtained from 3 experiments (4-well plate each) and presented as mean \pm standard error of mean (SEM). ANOVA and post-hoc Duncan's test were used to compare different groups using IBM SPSS statistics 22. $P<0.05$ was considered as statistically significant.

\section{Results \\ Effect of acrylamide on the survival of dopaminergic neurons}

As shown in Figure 1A, incubation of primary mesencephalic cell cultures with different concentrations of ACR $(0.001,0.01,0.1,1,2 \mathrm{mM})$ on the $9^{\text {th }}$ DIV for $24 \mathrm{~h}$ did not significantly affect the number of dopaminergic neurons. However, treatment of cultures with the same concentrations of ACR on the $9^{\text {th }}$ DIV for $48 \mathrm{~h}$ significantly decreased the number of dopaminergic neurons by $47.10 \%$ and $60.69 \%$ at the concentrations of 1 and $2 \mathrm{mM}$, respectively, compared to untreated controls (Fig. 1A). The $\mathrm{LC}_{50}$ of $A C R$ on dopaminergic neurons was calculated as $1 \mathrm{mM}$ in cultures treated with ACR on the $9^{\text {th }}$ DIV for $48 \mathrm{~h}$ (Fig. 1A). Moreover, when cultures were incubated with ACR for 24 or 48 h, it significantly decreased the number of dopaminergic neurites, respectively, by $17.16 \%$ and $19.53 \%$ at the concentration of $1 \mathrm{mM}$, and $38.32 \%$ and $53.09 \%$ at the concentration of $2 \mathrm{mM}$ compared to untreated controls (Fig. 1B).

\section{Effect of acrylamide on the morphology of dopaminergic neurons}

Untreated dopaminergic neurons were intact with long and branched processes after both 10 DIV (Fig. 2A,B) and 11 DIV (Fig. 3A,B). In 24 h-treated cultures, ACR was found to slightly affect dopaminergic neurons at the concentration of $1 \mathrm{mM}$ (Fig. 2C,D) and produced marked loss and shortening of dopaminergic neurites of surviving cells at the concentration $2 \mathrm{mM}$ (Fig. 2E,F). In 48 h-treated cultures, ACR treatment resulted in fewer dopaminergic neurons, and marked loss and shortening of dopaminergic neurites at both $1 \mathrm{mM}$ (Fig. 3C,D) and $2 \mathrm{mM}$ (Fig. 3E,F) concentrations. 

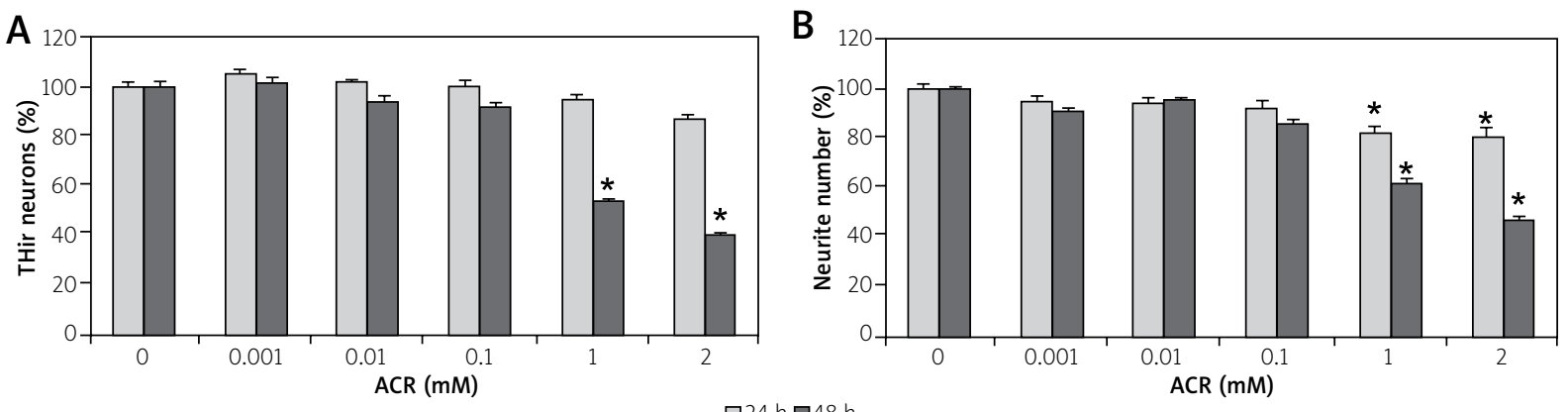

Fig. 1. Effect of acrylamide (ACR) on the survival of dopaminergic neurons in the primary mesencephalic cell culture. A) ACR did not produce any significant effect on the survival of dopaminergic neurons in $24 \mathrm{~h}$-treated cultures. On the other hand, ACR significantly decreased the number of dopaminergic neurons at the concentrations of $1(47.10 \pm 1.00)$ and $2 \mathrm{mM}(60.69 \pm 1.27)$ in 48 h-treated cultures compared to untreated controls. B) ACR significantly decreased the number of dopaminergic neurites at the concentrations of 1 and $2 \mathrm{mM}$ when cultures treated for both 24 and $48 \mathrm{~h}$ were compared to untreated controls. $100 \%$ corresponds to the total number of dopaminergic neurons and their neurites after 10 (the average number of dopaminergic neurons $=43.21 \pm 0.96$ and neurites $=3.21 \pm 0.65$ ) and 11 DIV (the average number of dopaminergic neurons $=47.51 \pm 1.32$ and neurites $=3.50 \pm 0.78)$ in untreated control cultures $(* p<0.001)$.

\section{Effect of acrylamide on lactate dehydrogenase release into the culture media}

Interestingly, $\mathrm{LC}_{50}$ of $\mathrm{ACR}$ did not affect $\mathrm{LDH}$ release into the culture medium and only produced significant release $(130.37 \%)$ at the concentration of $2 \mathrm{mM}$ after $48 \mathrm{~h}$ compared to untreated control culture (Fig. 4). ACR did not affect LDH release in the cultures treated for 24 h (Fig. 4).

\section{Effect of acrylamide on the total neuronal cells}

Treatment of primary mesencephalic cell cultures with different concentrations of ACR (0.001, $0.01,0.1,1,2 \mathrm{mM}$ ) significantly decreased NeuN immunoreactivity by $15.85 \%$ and $18.51 \%$ at the concentrations of 1 and $2 \mathrm{mM}$, respectively, after $48 \mathrm{~h}$ compared to untreated controls (Fig. 5A). Figure 5B showed that ACR at the concentrations of 1 and $2 \mathrm{mM}$ resulted in decreasing NeuN immunoreactivity, compared to untreated control cultures.

\section{Long-term effects of acrylamide on primary mesencephalic cell culture}

Incubation of cultures with lower concentrations of ACR $(0.01,0.05,0.1,0.5 \mathrm{mM})$ on the $6^{\text {th }}$ DIV for 5 consecutive days significantly decreased the number of dopaminergic neurons by $16.26 \%, 17.55 \%$ and $10.39 \%$ at the concentrations of $0.05,0.1$ and $0.5 \mathrm{mM}$, respectively, compared to untreated control cultures (Fig. 6A). Moreover, LDH levels in the culture medium was increased following treatment of cultures with ACR by $91.97 \%$ and $100.89 \%$ at the concentrations of 0.1 and $0.5 \mathrm{mM}$ compared to untreated control cultures (Fig. 6B).

\section{Discussion}

Exposure to environmental toxins is one of the four best-documented pan cellular factors besides age, genetic mutations and inflammation leading to increasing the risk of developing PD [28]. Acrylamide is a common chemical that is widely used for synthesis of polyacrylamide, treatment of drinking water and as cosmetic additives, and forms in many of high temperature prepared carbohydrate-rich foods [23]. Although ACR has been documented as a neurotoxic substance since the 1950s, no studies have shown its effects on dopaminergic neurons, the cells that synthesize the dopamine neurotransmitter and their loss leads to PD, yet. Therefore, the present study tried to demonstrate the effect of ACR on primary dopaminergic neurons in neuronal cell culture. Our results showed that ACR significantly decreased the survival of dopaminergic neurons with a $\mathrm{LC}_{50}$ of $1 \mathrm{mM}$ after $48 \mathrm{~h}$ of exposure. Our results are consistent with previous studies on other cellular and animal models. For instance, Li et al. [13] report- 

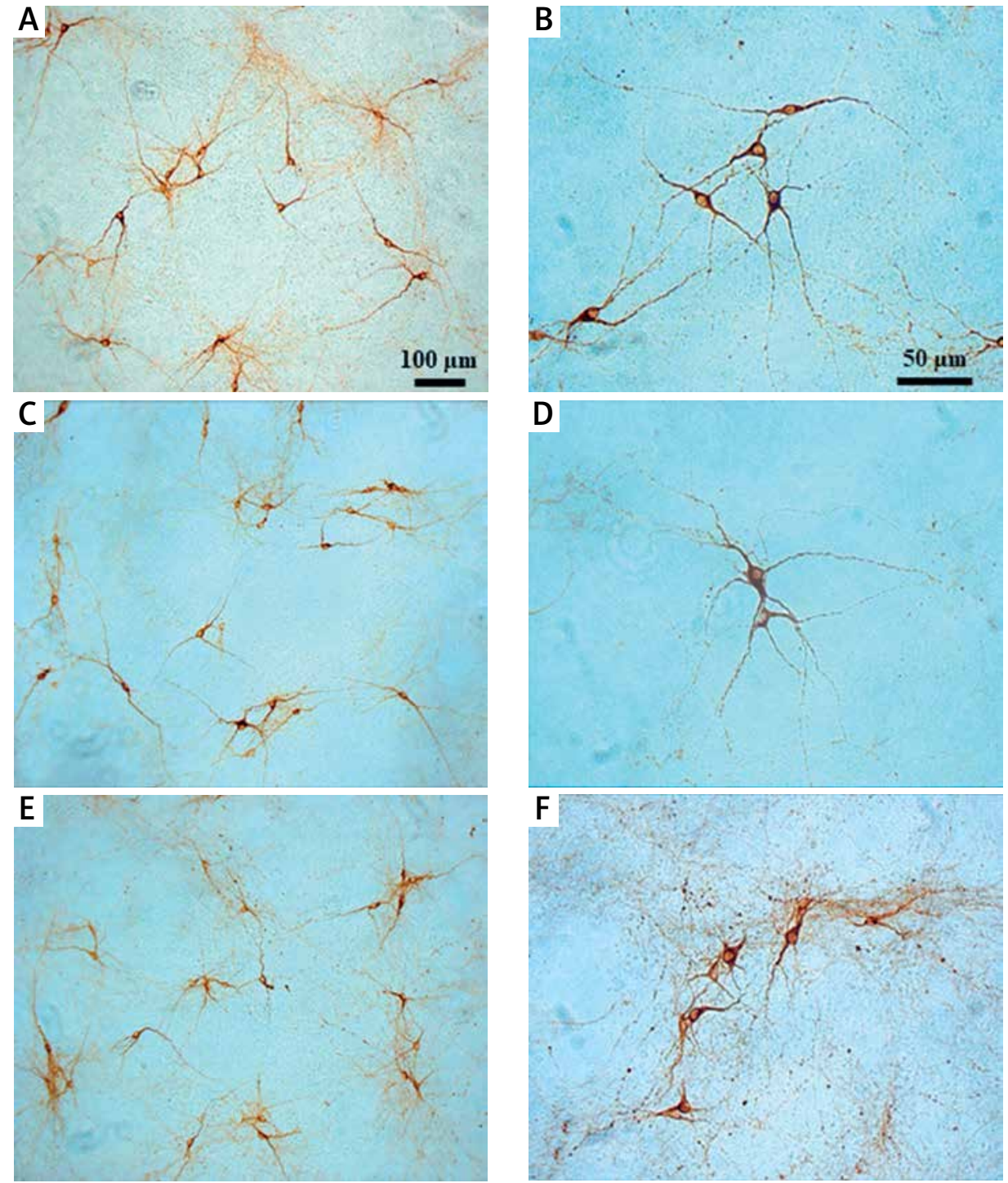

Fig. 2. Representative micrographs of dopaminergic neurons after 10 DIV. A, B) Untreated control cultures showed many and intact dopaminergic neurons with long and branched neurites. C, D) Acrylamide (ACR) at the concentration of $1 \mathrm{mM}$ slightly affects the morphology of dopaminergic neurons in $24 \mathrm{~h}$-treated cultures. E, F) Cultures treated with $2 \mathrm{mM}$ of ACR for $24 \mathrm{~h}$ showed a marked loss and shortening of dopaminergic neurites.

ed that ACR significantly decreased the viability of PC12 cells. Albalawi et al. [2] found that treatment of human retinal epithelial cells (RPE) with $1 \mathrm{mM}$ ACR for $24 \mathrm{~h}$ increased their cell death rate. Zhao et al. [30] showed that ACR at a concentration of $1 \mathrm{mM}$ adversely affected astrocytes and microglia in primary astrocytes/microglia co-culture. Li et al. [12] found that ACR was efficient in producing crucial Parkinsonian-like pathology including dopaminergic damage and $\alpha$-synuclein aggregation in C. elegans. In animal models, Abdelall et al. [1] and Lai et al. [10] reported ACR-induced progressive degeneration of Purkinje cells and hippocampal neurons in rats, respectively. Incubation of cultures with similar concentrations of ACR for $24 \mathrm{~h}$ did not significantly affect the number of dopaminergic neurons. Moreover, ACR was seen to adversely affect the morphology of dopaminergic neurons concomitant with a reduction in the numbers and lengths of dopaminergic neurites. Similarly, neurite outgrowth was reported to be more sensi- 

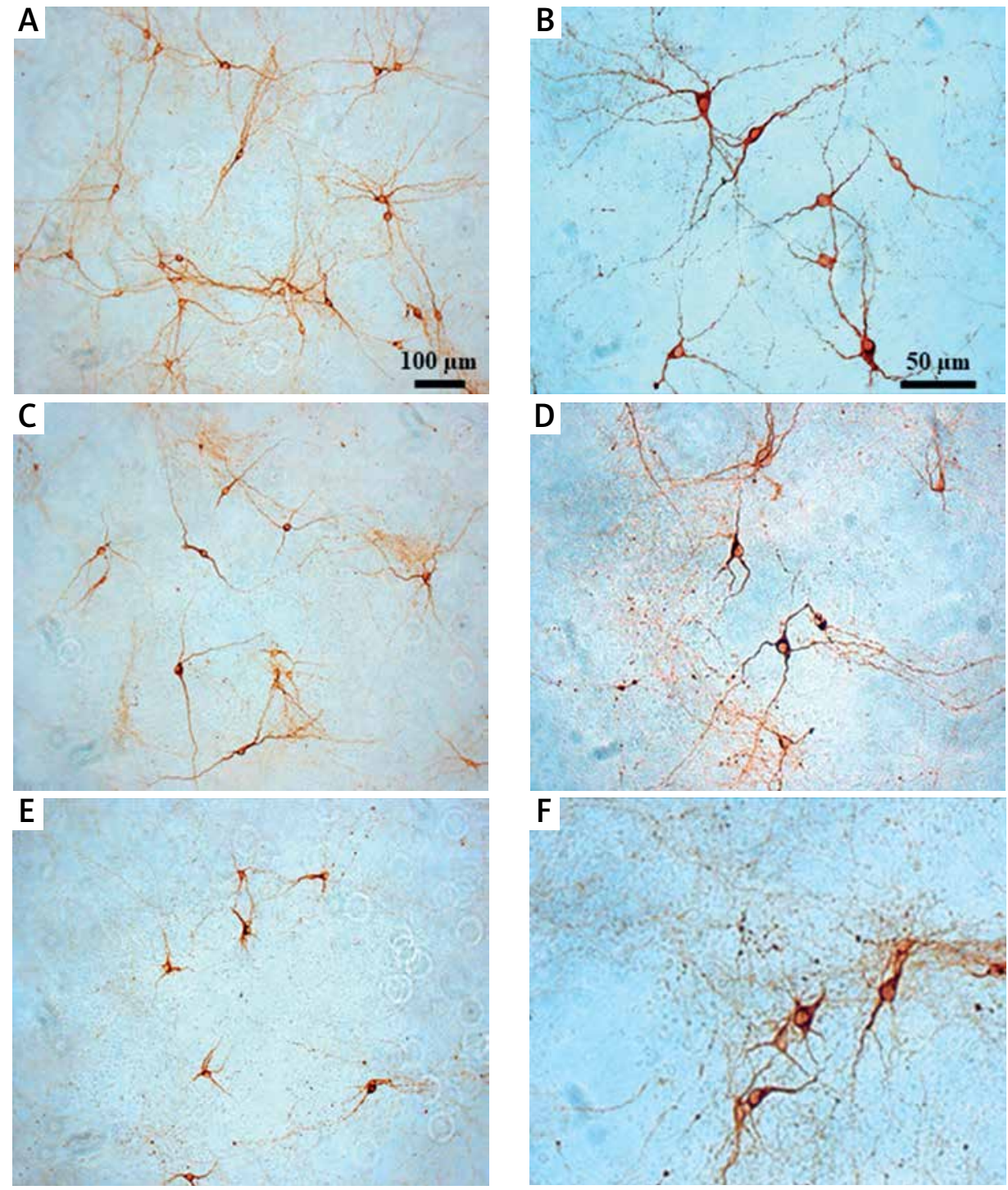

Fig. 3. Representative micrographs of dopaminergic neurons after 11 DIV. A, B) Untreated control cultures showed many and intact dopaminergic neurons with long and branched neurites. C, D) Treatment of cultures with $1 \mathrm{mM}$ of acrylamide (ACR) for $48 \mathrm{~h}$ resulted in a reduction in the number of dopaminergic neurons, and a marked loss and shortening of dopaminergic neurites. E, F) Cultures treated with $2 \mathrm{mM}$ of ACR showed a marked loss of dopaminergic neurons, and short and dysmorphic neurites.

tive to $A C R$ exposure in some in vitro studies. For example, Chen et al. [5] reported that exposure of NGF- or FGF-stimulated PC12 cells to $0.5 \mathrm{mM}$ of ACR significantly decreased their neurite outgrowth compared to untreated control cultures. Also, Attoff et al. [4] found that ACR decreased neurite number and impaired their outgrowth in differentiated SH-SY5Y cell line. Measurement of the neurite number and length (neurite outgrowth) is considered as a useful screening assay to identify potential developmental neurotoxicants [21]. In contrast to its toxic effect on dopaminergic neurons, $L_{50}$ of ACR had no effect on $\mathrm{LDH}$ release into the culture medium and only caused significant $L D H$ release at the concentration of $2 \mathrm{mM}$ compared to untreated control cultures. These results agree with some other studies reporting no significant effect of ACR on LDH activity [22,29]. Lack of release of $L D H$ by $L C_{50}$ of $A C R$ into culture medi- 


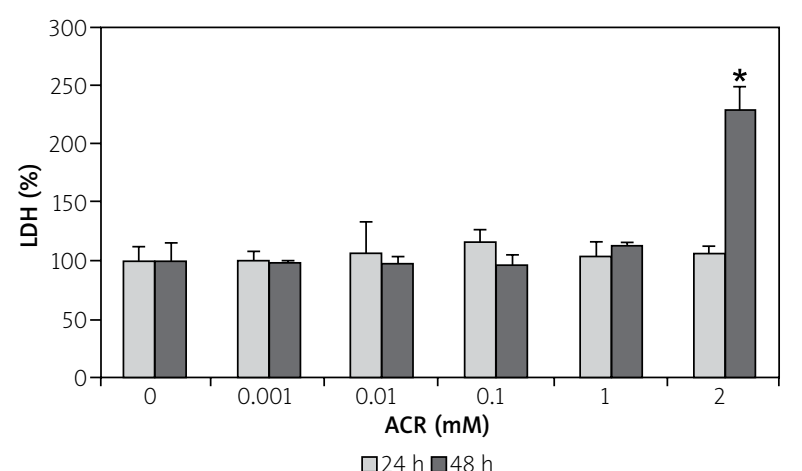

Fig. 4. Effects of acrylamide (ACR) on lactate dehydrogenase $(\mathrm{LDH})$ release into the culture medium. ACR significantly increased LDH release into the culture medium when cultures were treated with $2 \mathrm{mM}$ of ACR on the $9^{\text {th }}$ DIV for $48 \mathrm{~h}$ compared to untreated controls. $100 \%$ corresponds to the amount of LDH in the culture medium after 12 DIV $(* p<0.001)$. um indicates, to some extent, the selective effect on dopaminergic neurons and absence of overall toxicity on primary mesencephalic cell culture. Furthermore, ACR at both 1 and $2 \mathrm{mM}$ concentrations generated about a 15-18\% reduction in NeuN immunoreactivity indicating a decrease in the total neuronal cell populations in the culture. Likewise, ACR was reported to reduce the expression of the neuronal and astrocytes biomarkers in differentiating C17-2 cell cultures [4]. This slight effect of ACR on total neuronal cells in our primary mesencephalic cell cultures supports the idea of the absence of overall toxicity and provides a reason for insignificant $\mathrm{LDH}$ release into the culture medium. Acrylamide neurotoxicity in humans was reported to occur as a result of exposure to high doses in occupational settings [8]. However concerns about low levels of chronic exposure are increasing due to increasing the evidence of ACR cumulative effects [17,27]

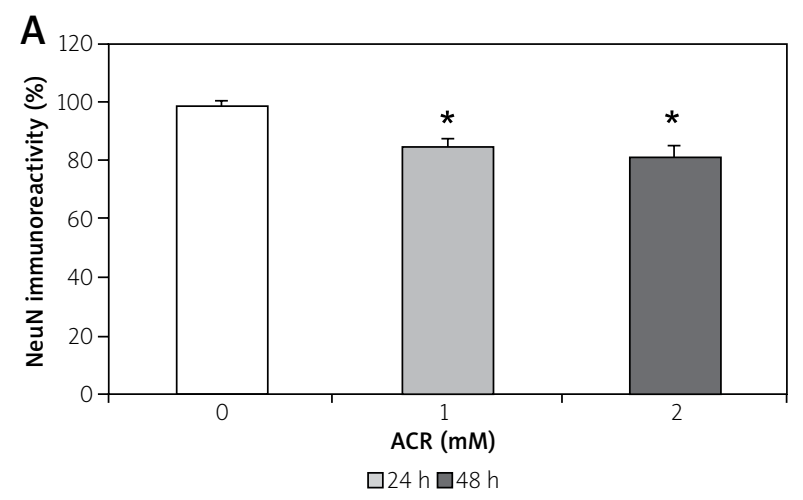

B
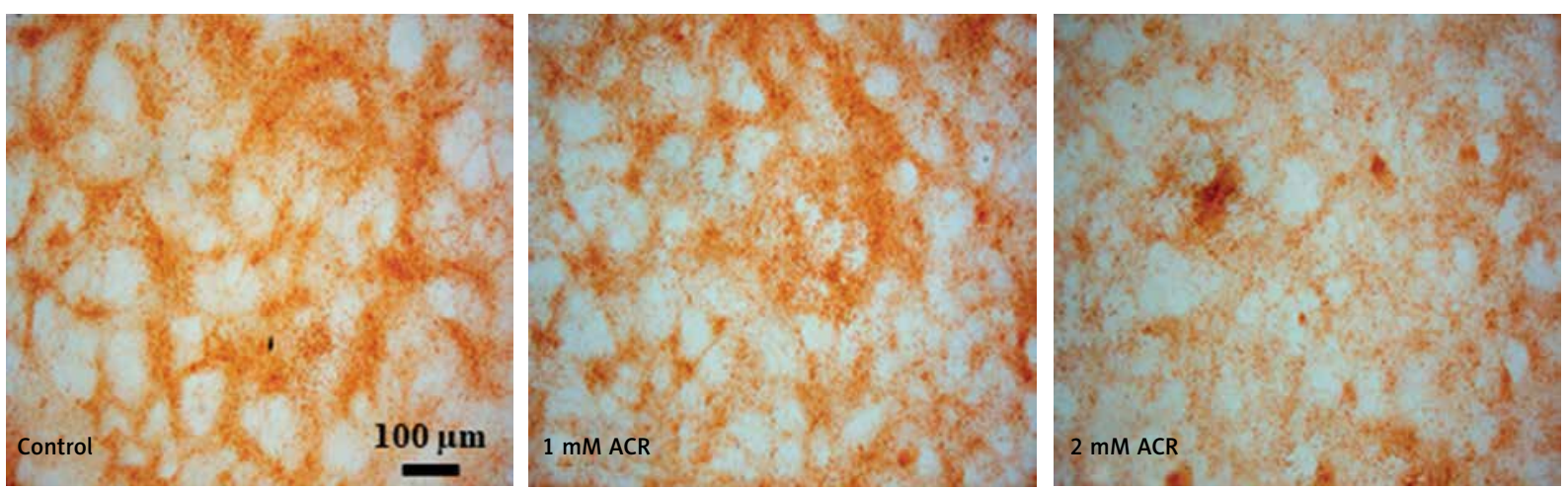

Fig. 5. Effect of acrylamide (ACR) on the neuronal nuclear antigen (NeuN) immunoreactivity in the primary mesencephalic cell culture. A) ACR significantly decreased NeuN immunoreactivity by $15.85 \pm 3.45 \%$ and $18.51 \pm 4.00 \%$ at the concentrations of 1 and $2 \mathrm{mM}$, respectively, after $48 \mathrm{~h}$ compared to untreated controls. $100 \%$ corresponds to NeuN immunoreactivity after 12 DIV in untreated control cultures $\left({ }^{*} p<0.001\right)$. B) Treatment of cultures with ACR for $48 \mathrm{~h}$ markedly decreased the expression of NeuN marker at the concentrations of 1 and $2 \mathrm{mM}$ compared to untreated control cultures. 

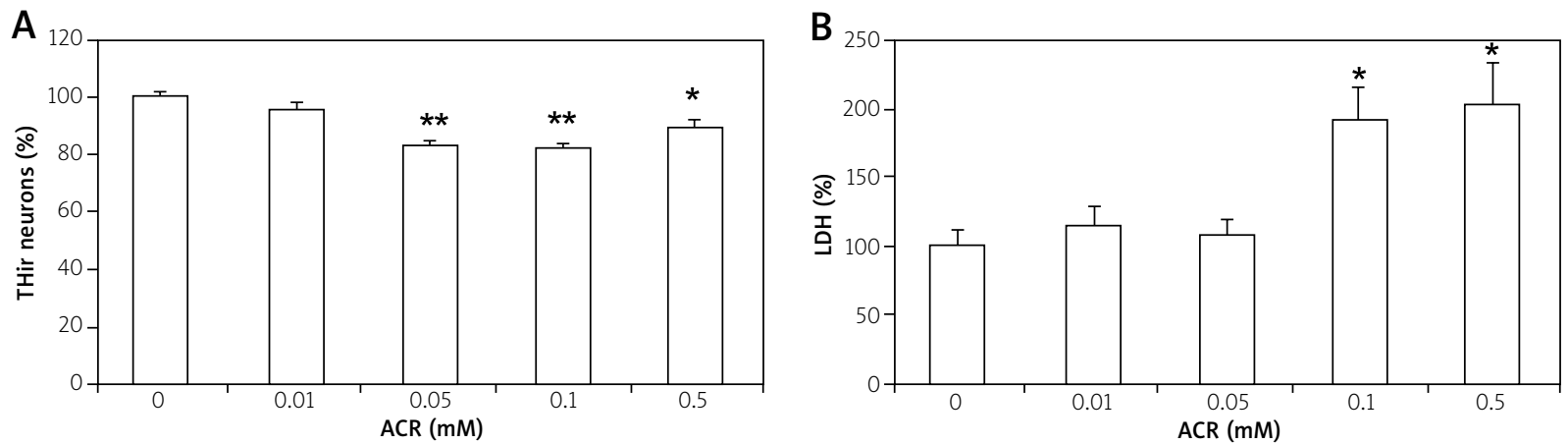

Fig. 6. Long-term effects of acrylamide (ACR) on the primary mesencephalic cell culture. A) Treatment of cultures with ACR for 5 consecutive days significantly decreased the number of dopaminergic neurons by $16.26 \pm 1.23 \%, 17.55 \pm 1.50 \%$ and $10.39 \pm 2.77 \%$ at the concentrations of $0.05,0.1$ and $0.5 \mathrm{mM}$, respectively, compared to untreated controls. B) Similar treatment of cultures with ACR significantly increased the release of lactate dehydrogenase (LDH) into the culture media by $91.97 \pm 23.61 \%$ and $100.89 \pm 32.11 \%$ at the concentrations of 0.1 and $0.5 \mathrm{mM}$, respectively, compared to untreated controls. $100 \%$ corresponds to the total number of dopaminergic neurons and the amount of LDH in the culture media after 11 DIV $\left({ }^{*} p<0.05,{ }^{* *} p<0.001\right)$.

and formation of ACR in high temperature-exposed starchy foods [2,24]. To investigate long-term effects of lower concentrations of ACR on dopaminergic neurons, a group of primary mesencephalic cell cultures was treated with ACR $(0.01,0.05,0.1,0.5 \mathrm{mM})$ on the $6^{\text {th }}$ DIV for 5 consecutive days. Our results showed that the concentrations of $0.05,0.1$ and $0.5 \mathrm{mM}$ decreased the dopaminergic cell number by about $15 \%$, and the concentrations of 0.1 and $0.5 \mathrm{mM}$ increased LDH release into the culture medium by about $100 \%$, compared to untreated control cultures. These concentrations did not affect both the number of dopaminergic neurons and the levels of $\mathrm{LDH}$ in the short-term regime where cultures were treated with ACR for $48 \mathrm{~h}$. This indicates that ACR may have some cumulative effects in the primary mesencephalic cell culture.

In conclusion, our current study reports, for the first time, the adverse effects of ACR on dopaminergic neurons in the primary mesencephalic cell culture.

\section{Acknowledgments}

The authors extend their appreciation to the Deanship of Scientific Research at King Khalid University for funding this work through the General Research Project under the grant number (KKUGRP-162-39).

\section{Disclosure}

The authors report no conflict of interest.

\section{References}

1. Abdelall HF, ElGhamrawy TA, Helmy D. Morphological evaluation of the protective role of dark soy sauce against acrylamide induced neurotoxicity in albino rats. Folia Morphol (Warsz) 2015; 74: 16-24.

2. Albalawi A, Alhasani RHA, Biswas L, Reilly J, Shu X. Protective effect of carnosic acid against acrylamide-induced toxicity in RPE cells. Food Chem Toxicol 2017; 108: 543-553.

3. Attoff K, Kertika D, Lundqvist J, Oredsson S, Forsby A. Acrylamide affects proliferation and differentiation of the neural progenitor cell line C17.2 and the neuroblastoma cell line SH-SY5Y. Toxicol In Vitro 2016; 35: 100-111.

4. Attoff K, Gliga A, Lundqvist J, Norinder U, Forsby A. Whole genome microarray analysis of neural progenitor C17.2 cells during differentiation and validation of 30 neural mRNA biomarkers for estimation of developmental neurotoxicity. PLoS One 2017; 12: e0190066.

5. Chen JH, Lee DC, Chiu IM. Cytotoxic effects of acrylamide in nerve growth factor or fibroblast growth factor 1-induced neurite outgrowth in PC12 cells. Arch Toxicol 2014; 88: 769-780.

6. Dauer W, Przedborski S. Parkinson's disease: mechanisms and models. Neuron 2003; 39: 889-909.

7. Erkekoglu P, Baydar T. Acrylamide neurotoxicity. Nutr Neurosci 2014; 17: 49-57.

8. Exon $\mathrm{JH}$. A review of the toxicology of acrylamide. J Toxicol Environ Health B Crit Rev 2006; 9: 397-412.

9. Kasabova-Angelova A, Tzankova D, Mitkov J, Georgieva M, Tzankova V, Zlatkov A, Kondeva-Burdina M. Xanthine deriva- 
tives as agents affecting non-dopaminergic neuroprotection in Parkinson's disease. Curr Med Chem 2018 [Epub ahead of print].

10. Lai SM, Gu ZT, Zhao MM, Li XX, Ma YX, Luo L, Liu J. Toxic effect of acrylamide on the development of hippocampal neurons of weaning rats. Neural Regen Res 2017; 12: 1648-1654.

11. Legrand C, Bour JM, Jacob C, Capiaumont J, Martial A, Marc A, Wudtke M, Kretzmer G, Demangel C, Duval D. Lactate dehydrogenase (LDH) activity of the number of dead cells in the medium of cultured eukaryotic cells as marker. J Biotech 1992; 25: 231-243.

12. Li J, Li D, Yang Y, Xu T, Li P, He D. Acrylamide induces locomotor defects and degeneration of dopaminergic neurons in C. elegans. J Appl Toxicol 2016; 36: 60-67.

13. Li L, Sun HY, Liu W, Zhao HY, Shao ML. Silymarin protects against acrylamide-induced neurotoxicity via Nrf2 signalling in PC12 cells. Food Chem Toxicol 2017; 102: 93-101.

14. Liu S, Jiang L, Zhong T, Kong S, Zheng R, Kong F, Zhang C, Zhang L, An L. Effect of acrylamide on oocyte nuclear maturation and cumulus cells apoptosis in mouse in vitro. PLoS One 2015; 10 : e0135818.

15. Marshall LJ, Willett C. Parkinson's disease research: adopting a more human perspective to accelerate advances. Drug Discov Today 2018; 3: 1950-1961.

16. Mehri S, Karami HV, Hassani FV, Hosseinzadeh H. Chrysin reduced acrylamide-induced neurotoxicity in both in vitro and in vivo assessments. Iran Biomed J 2014; 18: 101-106.

17. Pan X, Zhu L, Lu H, Wang D, Lu Q, Yan H. Melatonin attenuates oxidative damage induced by acrylamide in vitro and in vivo. Oxid Med Cell Longev 2015; 703709.

18. Pennisi M, Malaguarnera G, Puglisi V, Vinciguerra L, Vacante M, Malaguarnera M. Neurotoxicity of acrylamide in exposed workers. Int J Environ Res Public Health 2013; 10: 3843-3854.

19. Politi C, Ciccacci C, Novelli G, Borgiani P. Genetics and treatment response in Parkinson's disease: an update on pharmacogenetic studies. Neuromolecular Med 2018; 1: 1-17.

20. Radad K, Al-Shraim M, Al-Emam A, Moldzio R, Rausch WD. Neurotoxic effects of domoic acid on dopaminergic neurons in primary mesencephalic cell culture. Folia Neuropathol 2018; 56: 39-48.

21. Radio NM, Mundy WR. Developmental neurotoxicity testing in vitro: models for assessing chemical effects on neurite outgrowth. Neurotoxicology 2008; 29: 361-376.

22. Rajeh NA, Al-Dhaheri NM. Antioxidant effect of vitamin E and 5-aminosalicylic acid on acrylamide induced kidney injury in rats. Saudi Med J 2017; 38: 132-137.

23. Rivadeneyra-Domínguez E, Becerra-Contreras $Y$, Vázquez-Luna A, Díaz-Sobac R, Rodríguez-Landa JF. Alterations of blood chemistry, hepatic and renal function, and blood cytometry in acrylamide-treated rats. Toxicol Rep 2018; 5: 1124-1128.

24. Sansano M, Heredia A, Peinado I, Andrés A. Dietary acrylamide: What happens during digestion? Food Chem 2017; 237: 58-64.

25. Semla M, Goc Z, Martiniaková M, Omelka R, Formicki G. Acrylamide: a common food toxin related to physiological functions and health. Physiol Res 2017; 66: 205-217.

26. Sison SL, Vermilyea SC, Emborg ME, Ebert AD. Using Patient-Derived Induced Pluripotent Stem Cells to Identify Parkinson's
Disease-Relevant Phenotypes. Curr Neurol Neurosci Rep 2018; 18: 84 .

27. Spencer PS, Schaumburg HH. Nervous system degeneration produced by acrylamide monomer. Environ Health Perspect 1975; 11: 129-133.

28. Surmeier DH, Jaime N. Guzman JN, Sanchez-Padilla J, Goldberg JA. What causes the death of dopaminergic neurons in Parkinson's disease? Prog Brain Res 2010; 183: 59-77.

29. Yousef MI, El-Demerdash FM. Acrylamide-induced oxidative stress and biochemical perturbations in rats. Toxicology 2006; 219: 133-141.

30. Zhao M, Wang FSL, Hu XS, Chen F, Chan HM. Effect of acrylamide-induced neurotoxicity in a primary astrocytes/microglial co-culture model. Toxicol In Vitro 2017: 39: 119-125. 